\title{
Generalized nonlinear Proca equation and its free-particle solutions
}

\author{
F. D. Nobre ${ }^{1, a}$, A. R. Plastino ${ }^{2, b}$ \\ ${ }^{1}$ Centro Brasileiro de Pesquisas Físicas and National Institute of Science and Technology for Complex Systems, Rua Xavier Sigaud 150, \\ 22290-180 Rio de Janeiro, RJ, Brazil \\ ${ }^{2}$ CeBio y Secretaría de Investigación, Universidad Nacional Buenos Aires-Noreoeste, UNNOBA-Conicet, Roque Saenz Peña 456, Junín, Argentina
}

Received: 24 March 2016 / Accepted: 10 June 2016 / Published online: 21 June 2016

(C) The Author(s) 2016. This article is published with open access at Springerlink.com

\begin{abstract}
We introduce a nonlinear extension of Proca's field theory for massive vector (spin 1) bosons. The associated relativistic nonlinear wave equation is related to recently advanced nonlinear extensions of the Schrödinger, Dirac, and Klein-Gordon equations inspired on the non-extensive generalized thermostatistics. This is a theoretical framework that has been applied in recent years to several problems in nuclear and particle physics, gravitational physics, and quantum field theory. The nonlinear Proca equation investigated here has a power-law nonlinearity characterized by a real parameter $q$ (formally corresponding to the Tsallis entropic parameter) in such a way that the standard linear Proca wave equation is recovered in the limit $q \rightarrow 1$. We derive the nonlinear Proca equation from a Lagrangian, which, besides the usual vectorial field $\Psi^{\mu}(\vec{x}, t)$, involves an additional field $\Phi^{\mu}(\vec{x}, t)$. We obtain exact time-dependent soliton-like solutions for these fields having the form of a $q$-plane wave, and we show that both field equations lead to the relativistic energy-momentum relation $E^{2}=p^{2} c^{2}+m^{2} c^{4}$ for all values of $q$. This suggests that the present nonlinear theory constitutes a new field theoretical representation of particle dynamics. In the limit of massless particles the present $q$ generalized Proca theory reduces to Maxwell electromagnetism, and the $q$-plane waves yield localized, transverse solutions of Maxwell equations. Physical consequences and possible applications are discussed.
\end{abstract}

\section{Introduction}

The aim of the present contribution is to advance and explore some features of a nonlinear extension of Proca's field equation. This proposal is motivated by recent developments con-

\footnotetext{
a e-mail: fdnobre@cbpf.br

be-mail: arplastino@unnoba.edu.ar
}

cerning nonlinear extensions of the Schrödinger, Dirac, and Klein-Gordon equations [1-7], related to the non-extensive generalized thermostatistics $[8,9]$. These nonlinear equations are closely related to a family of power-law nonlinear Fokker-Planck equations that describe the spatio-temporal behavior of various physical systems and processes and have been studied intensively in recent years [10-17].

The Proca equation [18] constitutes, along with the Dirac and the Klein-Gordon equations, one of the fundamental relativistic wave equations [19]. It describes massive vector (spin 1) boson fields. Historically it played an important role due, among other things, to its relation with Yukawa's work on mesons [20]. Proca's equation also shields a generalization of Maxwell's electromagnetic field theory, by incorporating the effects of a finite rest mass for the photon [21]. As such, it is a basic ingredient of the theoretical framework for experimental studies aiming at the determination of upper bounds for the mass of the photon [22,23]. This is a basic line of enquiry that can be regarded as having its origins in experimental work by Cavendish and Maxwell, with their explorations of possible deviations from Coulomb's electrostatic force law [22] (although they, obviously, did not formulate this problem in terms of the mass of the photon). Within the modern approach, the Proca field equation allows for definite quantitative predictions concerning diverse physical effects originating in a finite mass of the photon (including the aforementioned deviations from Coulomb's law). This, in turn, motivates concrete experiments for the search of the mentioned effects, and for determining concomitant upper bounds for the photon's rest mass. Besides the problem of establishing bounds to the photon mass, the study of the Proca field equation has been a subject of constant interest in theoretical physics [24-29]. Proca-like modifications of electromagnetism have been considered in order to explore possible violations of Lorentz invariance at large distances [25]. The Proca equation with a negative square-mass constitutes a the- 
oretical tool for the analysis of tachyon physics [26]. Among other interesting aspects of the Proca field we can mention the rich variety of phenomena associated with the coupled Einstein-Proca field equations [28], which constitute a natural extension of the celebrated Einstein-Maxwell equations. Last, but certainly not least, the Proca field may be related to dark matter [29], whose nature is one of the most pressing open problems in contemporary science.

The nonlinear Schrödinger, Dirac, and Klein-Gordon equations investigated in [1-7] share the physically appealing property of admitting (in the case of vanishing interactions) exact soliton-like localized solutions that behave as free particles, in the sense of complying with the celebrated Einstein-Planck-de Broglie relations connecting frequency and wave number, respectively, with energy and momentum. Given these previous developments, it is natural to ask if the corresponding nonlinear extension can also be implemented for massive vector bosons. This is the question we are going to explore in the present contribution. Of especial relevance for our purposes is the nonlinear Klein-Gordon equation proposed in [1]. It exhibits a nonlinearity in the mass term which is proportional to a power of the wave function $\Phi(x, t)$. For the above mentioned exact solutions the wave function $\Phi(x, t)$ depends on space and time only through the combination $x-v t$. This space-time dependence corresponds to a uniform translation at a constant velocity $v$ without change in the shape of the wave function. These solitonlike solutions are called $q$-plane waves and, as already mentioned, are compatible with the Einstein-Planck-de Broglie relations $E=\hbar w$ and $p=\hbar k$, satisfying the relativistic energy-momentum relation $E^{2}=c^{2} p^{2}+m^{2} c^{4}$. The $q$-plane waves constitute a generalization of the standard exponential plane waves that arise naturally within a theoretical framework that extends the Boltzmann-Gibbs (BG) entropy and statistical mechanics on the basis of a power-law entropic functional $S_{q}$. This functional is parameterized by a real index $q$, the usual BG formalism being recovered in the limit $q \rightarrow$ 1 . The $q$-plane waves are complex-valued versions of the $q$-exponential distributions that optimize the $S_{q}$ entropies under appropriate constraints. These distributions are at the core of the alluded extension of the BG thermostatistics, which has been applied in recent years to a variegated set of physical scenarios. In particular, several applications to problems in nuclear and particle physics, as well as in quantum field theory, have been recently advanced. As examples we can mention applications of the $q$-non-extensive thermostatistics to the study of the nuclear equation of state [30-32], to neutron stars $[33,34]$, to the thermodynamics of hadron systems [35-38], to proton-proton and heavy ion collisions [39-45], to quantum cromodynamics [46-48], to cosmic rays [49], to the Thomas-Fermi model of self-gravitating systems [50], to fractal deformations of quantum statistics [51], and to the entropic-force approach to gravitation [52,53]. Intriguing connections between the non extensive thermostatistical formalism and $q$-deformed dynamics have been suggested [54]. The $q$-non-extensive thermostatistics has also stimulated the exploration of other non-additive entropic functionals that have been applied, for instance, to black hole thermodynamics $[55,56]$.

The paper is organized as follows. In Sect. 2 we introduce a Lagrangian for the generalized Proca field that leads to the nonlinear Proca field equations. We study some of its main properties, with special emphasis on the $q$-plane wave soliton-like solutions. In Sect. 3 we consider the limit of massless particles, obtaining a new family of wave-packet solutions of Maxwell equations. Some conclusions and final remarks are given in Sect. 4.

\section{Lagrangian approach for nonlinear Proca equations}

Let us introduce the four-dimensional space-time operators [19],

$\begin{aligned} & \partial^{\mu} \equiv \frac{\partial}{\partial x_{\mu}} \equiv\left\{\frac{\partial}{\partial(c t)},-\frac{\partial}{\partial x},-\frac{\partial}{\partial y},-\frac{\partial}{\partial z}\right\} ; \\ & \partial_{\mu} \equiv \frac{\partial}{\partial x^{\mu}} \equiv\left\{\frac{\partial}{\partial(c t)}, \frac{\partial}{\partial x}, \frac{\partial}{\partial y}, \frac{\partial}{\partial z}\right\},\end{aligned}$

as well as the contravariant and covariant vectors

$\Psi^{\mu} \equiv\left(\Psi_{0}, \Psi_{x}, \Psi_{y}, \Psi_{z}\right) ; \quad \Psi_{\mu} \equiv\left(\Psi_{0},-\Psi_{x},-\Psi_{y},-\Psi_{z}\right)$,

$\Phi^{\mu} \equiv\left(\Phi_{0}, \Phi_{x}, \Phi_{y}, \Phi_{z}\right) ; \quad \Phi_{\mu} \equiv\left(\Phi_{0},-\Phi_{x},-\Phi_{y},-\Phi_{z}\right)$.

As will be shown below, these two vector fields are necessary for a consistent field theory, similarly to the recent nonlinear versions of the Schrödinger and Klein-Gordon equations [2, 7]. For this purpose, we introduce the following Lagrangian density:

$$
\begin{aligned}
\mathcal{L}= & A\left\{-\frac{1}{2} F_{\mu \nu} \tilde{F}^{\mu \nu}+q \frac{m^{2} c^{2}}{\hbar^{2}}\left(\Phi_{\mu} \Psi^{\mu}\right)\left(\Psi_{\nu} \Psi^{\nu}\right)^{q-1}\right. \\
& \left.-\frac{1}{2} F_{\mu \nu}^{*} \tilde{F}^{\mu \nu *}+! q \frac{m^{2} c^{2}}{\hbar^{2}}\left(\Phi_{\mu}^{*} \Psi^{\mu *}\right)\left(\Psi_{\nu}^{*} \Psi^{\nu *}\right)^{q-1}\right\},
\end{aligned}
$$

where $A$ is an appropriate multiplicative factor that may depend on the total volume (in the case the fields are confined in a finite volume $V$ ). Moreover, we are adopting the standard index-summation convention [19], and the tensors above are given by

$F_{\mu \nu}=\partial_{\mu} \Phi_{\nu}-\partial_{\nu} \Phi_{\mu} ; \quad \tilde{F}^{\mu \nu}=\partial^{\mu} \Psi^{\nu}-\partial^{\nu} \Psi^{\mu}$.

The Euler-Lagrange equations for the vector field $\Phi_{\mu}[19]$,

$$
\frac{\partial \mathcal{L}}{\partial \Phi_{\mu}}-\partial_{\nu}\left[\frac{\partial \mathcal{L}}{\partial\left(\partial_{\nu} \Phi_{\mu}\right)}\right]=0
$$


lead to nonlinear Proca field equations,

$$
\begin{gathered}
\frac{1}{c^{2}} \frac{\partial^{2} \Psi^{\mu}}{\partial t^{2}}-\nabla^{2} \Psi^{\mu}-\partial^{\mu}\left(\partial_{\nu} \Psi^{\nu}\right) \\
+q \frac{m^{2} c^{2}}{\hbar^{2}} \Psi^{\mu}\left(\Psi_{\nu} \Psi^{\nu}\right)^{q-1}=0,
\end{gathered}
$$

which implies the nonlinear subsidiary condition $\partial_{\mu}\left[\Psi^{\mu}\right.$ $\left.\left(\Psi_{\nu} \Psi^{\nu}\right)^{q-1}\right]=0$. We are going to prove the existence of, and pay special attention to, solutions of the field equations (7) that also comply with the Lorentz condition $\partial_{v} \Psi^{v}=$ $0[19,21]$. The Euler-Lagrange equations for the vector field $\Psi_{\mu}$,

$$
\frac{\partial \mathcal{L}}{\partial \Psi_{\mu}}-\partial_{\nu}\left[\frac{\partial \mathcal{L}}{\partial\left(\partial_{\nu} \Psi_{\mu}\right)}\right]=0
$$

yield

$$
\begin{gathered}
\frac{1}{c^{2}} \frac{\partial^{2} \Phi_{\mu}}{\partial t^{2}}-\nabla^{2} \Phi_{\mu}-\partial_{\mu}\left(\partial^{\nu} \Psi_{\nu}\right)+q \frac{m^{2} c^{2}}{\hbar^{2}}\left[\Phi_{\mu}\left(\Psi_{\nu} \Psi^{\nu}\right)^{q-1}\right. \\
\left.+2(q-1) \Psi_{\mu}\left(\Phi_{\nu} \Psi^{\nu}\right)\left(\Psi_{\nu} \Psi^{\nu}\right)^{q-2}\right]=0 .
\end{gathered}
$$

One should notice that in the limit $q=1$, the field equations (7)-(9) imply the Lorentz subsidiary condition and thus reduce to the standard linear Proca equations [18,19,21], with $\Phi^{\mu}(\vec{x}, t)=\Psi^{\mu *}(\vec{x}, t)$. It is also worth to consider how the field equations (7) and (9) are respectively connected with the nonlinear Klein-Gordon equation

$$
\nabla^{2} \Psi(\vec{x}, t)=\frac{1}{c^{2}} \frac{\partial^{2} \Psi(\vec{x}, t)}{\partial t^{2}}+q \frac{m^{2} c^{2}}{\hbar^{2}}[\Psi(\vec{x}, t)]^{2 q-1} .
$$

and the associated equation

$\nabla^{2} \Phi(\vec{x}, t)=\frac{1}{c^{2}} \frac{\partial^{2} \Phi(\vec{x}, t)}{\partial t^{2}}+q(2 q-1) \frac{m^{2} c^{2}}{\hbar^{2}} \Phi(\vec{x}, t)[\Psi(\vec{x}, t)]^{2(q-1)}$,

recently introduced in Refs. [1,7]. In the case of the linear Proca equations, each component of the Proca field satisfy an independent differential equation having the form of the Klein-Gordon one. In the present $q$-generalized, nonlinear scenario, the situation is more complex. In contrast to what happens with the standard linear Proca equation, the four equations appearing both in (7) and in (9), besides being nonlinear, are coupled with each other. There is, however, a clear structural similarity between the Eqs. (10) and (11) and the Eqs. (7) and (9). The nonlinear Klein-Gordon equation can be regarded as a scalar (one component) version of the vectorial (four-component) nonlinear Proca equations.

For general values of the $q$ parameter, the fields $\Psi^{\mu}(\vec{x}, t)$ and $\Phi^{\mu}(\vec{x}, t)$ are distinct, and the solutions of Eqs. (7) and (9) may be written in terms of a $q$-plane wave, similarly to the recent nonlinear proposals of quantum equations $[1,2,7]$. In fact, one has

$$
\begin{aligned}
& \Psi^{\mu}(\vec{x}, t)=a^{\mu} \exp _{q}\left[\frac{i}{\hbar}(\vec{p} \cdot \vec{x}-E t)\right] \\
& \Phi^{\mu}(\vec{x}, t)=a^{\mu}\left\{\exp _{q}\left[\frac{i}{\hbar}(\vec{p} \cdot \vec{x}-E t)\right]\right\}^{-(2 q-1)},
\end{aligned}
$$

which satisfy Eqs. (7) and (9), provided that the coefficients satisfy the relations $a_{\mu} a^{\mu}=1$ and $(1 / \hbar)\left(a^{0} E-a^{1} p_{x}-\right.$ $\left.a^{2} p_{y}-a^{3} p_{z}\right)=0$. Under these constraints the $q$-plane waves (12) and (13) constitute exact analytical solutions of the field equations (7) and (9) complying with both the Lorentz and the nonlinear subsidiary conditions.

The solutions above are expressed in terms of the $q$ exponential function $\exp _{q}(u)$ that emerges in non-extensive statistical mechanics [8], which generalizes the standard exponential, and for a pure imaginary $i u$, it is defined as the principal value of

$\exp _{q}(i u)=[1+(1-q) i u]^{\frac{1}{1-q}} ; \quad \exp _{1}(i u) \equiv \exp (i u)$,

where we used $\lim _{\epsilon \rightarrow 0}(1+\epsilon)^{1 / \epsilon}=e$. The real and imaginary parts of $\exp _{q}(i u)$ are respectively given by the functions $\cos _{q} u$ and $\sin _{q} u$ (see Sect. 3 for more details). The dependence of the $q$-plane waves on the space-time coordinates is illustrated in Figs. 1 and 2, where the real and imaginary parts of the complex-valued function $\exp _{q}(i u)$ are depicted as a function of $u=\frac{1}{\hbar}(\vec{p} \cdot \vec{x}-E t)$.

Considering the $q$-plane wave solutions one obtains the energy-momentum relation

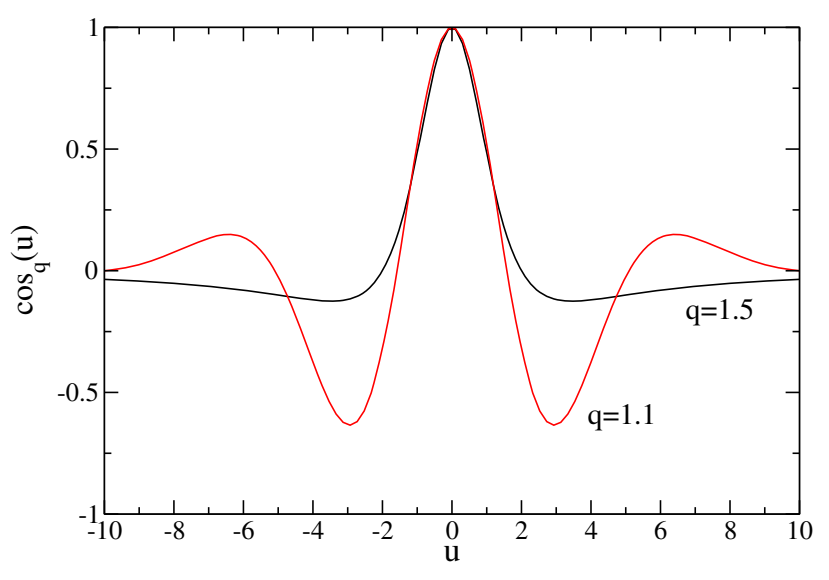

Fig. 1 Plot for two different values of $q$ of the real part of the complexvalued function $\exp _{q}(i u)$, given by the function $\cos _{q}(u)$. The spacetime dependence of the $q$-plane waves are given by $\exp _{q}(i u)=$ $\cos _{q}(u)+i \sin _{q}(u)$, where $u=\frac{1}{\hbar}(\vec{p} \cdot \vec{x}-E t)$. All depicted quantities are dimensionless 


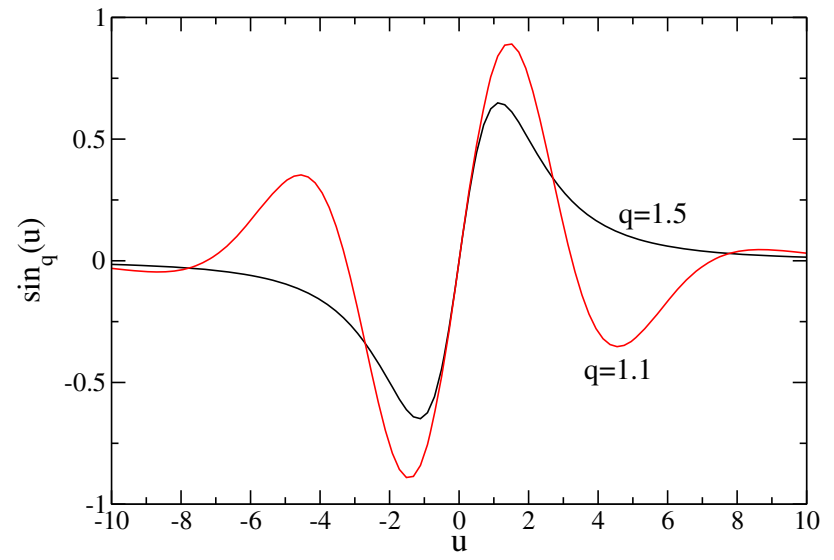

Fig. 2 Plot for two different values of $q$ of the imaginary part of the complex-valued function $\exp _{q}(i u)$, given by the function $\sin _{q}(u)$. All depicted quantities are dimensionless

$E^{2}=p^{2} c^{2}+m^{2} c^{4}$

from both Eqs. (7) and (9), for all $q$. Note that, as already mentioned and in contrast to what happens with the standard linear Proca equation, the four equations appearing both in (7) and in (9), besides being nonlinear, are coupled. It is remarkable that these sets of four nonlinear coupled partial differential equations admit exact time-dependent solutions of the $q$-plane wave form that are consistent with the relativistic energy-momentum equation (15).

When considering the nonlinear Proca theory based upon the field Eqs. (7) and (9), a comment concerning degrees of freedom is in order. It may seem that, since the present nonlinear generalization involves two fields, it is endowed with more degrees of freedom than the standard linear version of Proca equations. This, however, is not necessarily the case. The partial differential equation (7) for the evolution of the field $\Psi^{\mu}$ is autonomous. Given appropriate initial or boundary conditions for $\Psi^{\mu}$ the solution of Eq. (7) is fully determined: this equation can be solved without any reference to the other field $\Phi^{\mu}$. On the other hand, the evolution Eq. (9) for the field $\Phi^{\mu}$ explicitly involves the first field $\Psi^{\mu}$. We can say, figuratively speaking, that the field $\Psi^{\mu}$ is a leader field, while $\Phi^{\mu}$ can be regarded as a slave field. Given one solution for the field $\Psi^{\mu}$, there are infinite many possible solutions for the field $\Phi^{\mu}$ (all determined by $\Psi^{\mu}$ through Eq. (9)). We conjecture that, among all these possible solutions for the field $\Phi^{\mu}$, there is a particular one that is physically relevant, this special solution exhibiting a functional relation with the field $\Psi^{\mu}$, which effectively reduces the relevant degrees of freedom of the system solely to those associated with the field $\Psi^{\mu}$. This is what happens in the case of the $q$-plane wave solutions investigated in the present work, for which we have $\Phi^{\mu} \propto\left[\Psi^{\mu}\right]^{-(2 q-1)}$. In the $q \rightarrow 1$ limit the two fields are of course also related, since one has $\Phi^{\mu}=\Psi^{\mu *}$. It is worth stressing that for the $q$-plane wave solutions of the nonlinear field equations, the above mentioned power-law relation between the fields $\Phi^{\mu}$ and $\Psi^{\mu}$ reduces, in the $q \rightarrow 1$ limit, to the complex conjugation relation (since for standard complex exponential plane waves one has $\left[\Psi^{\mu}\right]^{-1}=\Psi^{\mu *}$ ).

We now consider the question of how to define a density associated with the fields of the nonlinear Proca theory. It is worth to recall here that, even in the linear case, the definition of probability densities associated with relativistic wave equations is highly problematic. For instance, the density associated with the standard Klein-Gordon equation does not have a definite sign: it can admit negative values. Consequently, even for the linear Klein-Gordon equation a probability interpretation is not feasible. One of the reasons behind this kind of difficulties is that the relativistic wave equations, on their own, are only the equations of classical field theories. In the case of the present nonlinear Proca dynamics a tentative definition of a density is given by

$\rho(\vec{x}, t)=\frac{1}{2}\left(\Psi^{v} F_{0 v}-\tilde{F}_{0}^{v} \Phi_{\nu}+\Psi^{\nu *} F_{0 v}^{*}-\tilde{F}_{0}^{\nu *} \Phi_{v}^{*}\right)$.

The heuristic motivation behind this proposal is that in the $q \rightarrow 1$ limit the density (16) reduces to the one associated with the standard linear Proca field [19]. Combining Eq. (16) with the field equations (7) and (9) it can be verified that (16) verifies a balance equation equivalent to a continuity equation plus an extra source term (see [5] for a discussion of a similar situation in connection with the $q$-generalized nonlinear Schrödinger equation introduced in [1]). The balance equation is associated with the current,

$J_{\mu}=\frac{1}{2}\left(\Psi^{\nu} F_{\mu \nu}-\tilde{F}_{\mu}^{v} \Phi_{\nu}+\Psi^{\nu *} F_{\mu \nu}^{*}-\tilde{F}_{\mu}^{\nu *} \Phi_{\nu}^{*}\right)$,

which, again, reduces to the conserved current for the linear Proca equation in the $q \rightarrow 1$ limit [19]. However, the current (17) is not conserved. That is, instead of the current conservation condition $\partial^{\mu} J_{\mu}=0$ one has the balance equation $\partial^{\mu} J_{\mu}=R \neq 0$, where $R$ is the aforementioned non-vanishing source term (which in general depends on the values of the fields and their space-time derivatives). In the particular case of the $q$-plane wave solutions (12) and (13) it is verified that the probability density (16) is conserved globally, in the sense that $\frac{\mathrm{d}}{\mathrm{d} t} \int \rho \mathrm{d}^{3} x=0$.

\section{Massless particles: $q$-plane waves as solutions of maxwell equations}

The linear Proca equations are usually considered as appropriate for describing vectorial bosons, or massive photons $[19,21]$. Now, if one considers the limit $m \rightarrow 0$ in the 
Lagrangian of Eq. (4), one eliminates its nonlinear contributions, recovering the electromagnetic Lagrangian without sources [21]. In this case, both Eqs. (7) and (9) reduce to the standard linear wave equation, described in terms of a single vector field (i.e., $\left.\Phi^{\mu}(\vec{x}, t)=\Psi^{\mu *}(\vec{x}, t)\right)$,

$\nabla^{2} \Psi^{\mu}=\frac{1}{c^{2}} \frac{\partial^{2} \Psi^{\mu}}{\partial t^{2}}$

As usual, considering $\vec{p}=\hbar \vec{k}$ and $E=\hbar \omega$, one verifies easily that any twice-differentiable function of the type $f(\vec{k}$. $\vec{x}-\omega t$ ) is a solution of the wave equation. In the following we will explore the $q$-plane wave of Eq. (12) as such a solution; our analysis is based on some properties of this solution, which are relevant from the physical point of view: (1) it presents an oscillatory behavior; (2) it is localized for certain values of $q$. Indeed, for $q \neq 1$ the $q$-exponential $\exp _{q}(i u)$ is characterized by an amplitude $r_{q} \neq 1$ [57],

$\exp _{q}( \pm i u)=\cos _{q}(u) \pm i \sin _{q}(u)$,

$\cos _{q}(u)=r_{q}(u) \cos \left\{\frac{1}{q-1} \arctan [(q-1) u]\right\}$,

$\sin _{q}(u)=r_{q}(u) \sin \left\{\frac{1}{q-1} \arctan [(q-1) u]\right\}$,

$r_{q}(u)=\left[1+(1-q)^{2} u^{2}\right]^{1 /[2(1-q)]}$,

so that $r_{q}(u)$ decreases for increasing arguments, if $q>1$.

From Eqs. (19)-(22) one notices that $\cos _{q}(u)$ and $\sin _{q}(u)$ cannot be zero simultaneously (even though their moduli do tend to zero simultaneously as $u \rightarrow \pm \infty$ ) yielding $\exp _{q}( \pm i u) \neq 0$; moreover, $\exp _{q}(i u)$ presents further peculiar properties,

$$
\begin{aligned}
& {\left[\exp _{q}(i u)\right]^{*}=\exp _{q}(-i u)=[1-(1-q) i u]^{\frac{1}{1-q}},} \\
& \exp _{q}(i u)\left[\exp _{q}(i u)\right]^{*}=\left[r_{q}(u)\right]^{2}=\left[1+(1-q)^{2} u^{2}\right]^{\frac{1}{1-q}}
\end{aligned}
$$

$\exp _{q}\left(i u_{1}\right) \exp _{q}\left(i u_{2}\right)=\exp _{q}\left[i u_{1}+i u_{2}-(1-q) u_{1} u_{2}\right]$,

$\left\{\left[\exp _{q}(i u)\right]^{\alpha}\right\}^{*}=\left\{\left[\exp _{q}(i u)\right]^{*}\right\}^{\alpha}=\left[\exp _{q}(-i u)\right]^{\alpha}$,

for any $\alpha$ real. By integrating Eq. (24) from $-\infty$ to $+\infty$, one obtains [58],

$\mathcal{I}_{q}=\int_{-\infty}^{\infty} \mathrm{d} u\left[r_{q}(u)\right]^{2}=\frac{\sqrt{\pi} \Gamma\left(\frac{3-q}{2(q-1)}\right)}{(q-1) \Gamma\left(\frac{1}{q-1}\right)}$,

leading to the physically important property of square integrability for $1<q<3$; as some simple typical examples, one has $\mathcal{I}_{3 / 2}=\mathcal{I}_{2}=\pi$. One should notice that this integral diverges in both limits $q \rightarrow 1$ and $q \rightarrow 3$, as well as for any $q<1$. Hence, the $q$-plane wave of Eq. (12) presents a modulation, typical of a localized wave, for $1<q<3$.

Then, identifying the components of the vector of Eq. (2) with the scalar and vector potentials, $\Psi^{\mu} \equiv(\phi, \vec{A})$, from which one obtains the fields [21],

$\vec{E}=-\vec{\nabla} \phi-\frac{1}{c} \frac{\partial \vec{A}}{\partial t} ; \quad \vec{B}=\vec{\nabla} \times \vec{A}$,

one verifies that the wave equations for these potentials are equivalent to the Maxwell equations in the absence of sources. When dealing with Maxwell equations the physically meaningful solutions of the field equations are the real solutions. However, as is well known, it is usually computationally convenient to consider complex solutions, since the linearity of the field equations imply that the real (or the imaginary) parts of those solutions are themselves also solutions of Maxwell equations. In the following we are going to obtain first the complex $q$-plane wave solutions that Maxwell equations inherit from the nonlinear $q$-generalized Proca equation, and them focus on their real parts.

Hence, we now consider the following solutions for each Cartesian component $j(j=x, y, z)$ of the electromagnetic fields:

$E_{j}(\vec{x}, t)=E_{0 j} \exp _{q}[i(\vec{k} \cdot \vec{x}-\omega t)] ;$

$B_{j}(\vec{x}, t)=B_{0 j} \exp _{q}[i(\vec{k} \cdot \vec{x}-\omega t)]$,

which satisfy the wave equation, for each component, provided that $\omega=c|\vec{k}|$. Writing the wave vector as $\vec{k}=k \vec{n}$, where $\vec{n}$ represents a unit vector along the wave-propagation direction, the Maxwell equations associated with the divergence of the fields $\vec{E}$ and $\vec{B}$ yield respectively,

$\vec{n} \cdot \vec{E}_{0}=0 ; \quad \vec{n} \cdot \vec{B}_{0}=0$,

implying that the fields $\vec{E}$ and $\vec{B}$ are both perpendicular to the direction of propagation. In addition to this, considering the solutions of (29) in Faraday's Law,

$\vec{\nabla} \times \vec{E}+\frac{1}{c} \frac{\partial \vec{B}}{\partial t}=0 \Rightarrow \vec{B}_{0}=\vec{n} \times \vec{E}_{0}$,

whereas doing the same in Ampère's Law,

$\vec{\nabla} \times \vec{B}-\frac{1}{c} \frac{\partial \vec{E}}{\partial t}=0 \Rightarrow \vec{E}_{0}=-\vec{n} \times \vec{B}_{0}$.

The results above show that an electromagnetic wave defined by (29) corresponds to a transverse wave, similarly to the plane-wave solution. Now, focusing on the particular case where the vectors $\vec{E}_{0}$ and $\vec{B}_{0}$ (which comply with the relations (31) and (32)) are real, the real parts of complex solutions 
(29) are given by

$$
\begin{aligned}
E_{j}= & E_{0 j}\left[1+(1-q)^{2}(\vec{k} \cdot \vec{x}-\omega t)^{2}\right]^{1 /[2(1-q)]} \\
& \times \cos \left\{\frac{1}{q-1} \arctan [(q-1)(\vec{k} \cdot \vec{x}-\omega t)]\right\} \\
B_{j}= & B_{0 j}\left[1+(1-q)^{2}(\vec{k} \cdot \vec{x}-\omega t)^{2}\right]^{1 /[2(1-q)]} \\
& \times \cos \left\{\frac{1}{q-1} \arctan [(q-1)(\vec{k} \cdot \vec{x}-\omega t)]\right\}
\end{aligned}
$$

The above equations provide real traveling wave solutions to Maxwell equations in vacuum associated with the $q$-plane wave solutions discussed before. From now on we shall refer to these solutions as real $q$-plane waves. For $q=2$ these solutions adopt a specially simple Lorentz-like form,

$E_{j}=E_{0 j}\left[1+(\vec{k} \cdot \vec{x}-\omega t)^{2}\right]^{-1}$,
$B_{j}=B_{0 j}\left[1+(\vec{k} \cdot \vec{x}-\omega t)^{2}\right]^{-1}$.

Let us consider now the associated Poynting vector,

$$
\begin{aligned}
\vec{S}= & \frac{c}{4 \pi}(\vec{E} \times \vec{B}) \\
= & \frac{c}{4 \pi}\left|\vec{E}_{0}\right|^{2}\left[1+(1-q)^{2}(\vec{k} \cdot \vec{x}-\omega t)^{2}\right]^{\frac{1}{1-q}} \\
& \times \cos ^{2}\left\{\frac{1}{q-1} \arctan [(q-1)(\vec{k} \cdot \vec{x}-\omega t)]\right\} \vec{n},
\end{aligned}
$$

as well as the corresponding energy density,

$$
\begin{aligned}
\mathcal{U}= & \frac{1}{8 \pi}(\vec{E} \cdot \vec{E}+\vec{B} \cdot \vec{B}) \\
= & \frac{1}{4 \pi}\left|\vec{E}_{0}\right|^{2}\left[1+(1-q)^{2}(\vec{k} \cdot \vec{x}-\omega t)^{2}\right]^{\frac{1}{1-q}} \\
& \times \cos ^{2}\left\{\frac{1}{q-1} \arctan [(q-1)(\vec{k} \cdot \vec{x}-\omega t)]\right\} .
\end{aligned}
$$

It transpires from the two expressions above that there is an important difference between the real $q$-plane wave solutions (33) and the standard plane wave solutions [21]: the energy density and the modulus of the Poynting vector are respectively upper-bounded by the enveloping functions $\Gamma$ and $c \Gamma$, where the factor $\Gamma=\frac{1}{4 \pi}\left|\vec{E}_{0}\right|^{2}\left[1+(1-q)^{2}(\vec{k} \cdot \vec{x}-\omega t)^{2}\right]^{\frac{1}{1-q}}$ is essentially a $q$-Gaussian of the argument $|\vec{k} \cdot \vec{x}-\omega t|$. One verifies that

$$
\begin{aligned}
\left(\frac{\partial \Gamma}{\partial t}\right)_{\vec{x}}= & \frac{1}{4 \pi}\left|\vec{E}_{0}\right|^{2}\left[1+(1-q)^{2}(\vec{k} \cdot \vec{x}-\omega t)^{2}\right]^{\frac{q}{1-q}} \\
& \times(q-1) \omega(\vec{k} \cdot \vec{x}-\omega t),
\end{aligned}
$$

which leads to $(\partial \Gamma / \partial t)_{\vec{x}}<0$, for $q>1$ if $\vec{k} \cdot \vec{x}<\omega t$. This result is directly related with the fact that the amplitude of the enveloping function $\Gamma$ decreases for increasing arguments, for $q>1$, according to Eqs. (19)-(22). Notice that $(\partial \Gamma / \partial t)_{\vec{x}}=0$ for $q=1$, as a consequence of the fact that the standard plane wave fills the whole space.

\subsection{Physical application: a real $q$-plane wave in a waveguide}

Let us now consider the propagation of a real $q$-plane wave in an infinite rectangular waveguide, adjusted appropriately along the wave-propagation direction, which will be chosen herein to be the $\vec{x}$ axis, i.e., $\vec{k} \cdot \vec{x}=k_{x} x$. The total energy $U$ carried by the real $q$-plane wave can be calculated from Eq. (36), leading to the inequality

$$
\begin{aligned}
U & =\int \mathrm{d} \vec{x} \mathcal{U}(\vec{x}, t) \\
& \leq \frac{\sigma}{4 \pi}\left|\vec{E}_{0}\right|^{2} \int_{-\infty}^{\infty} \mathrm{d} x\left[1+(1-q)^{2}\left(k_{x} x-\omega t\right)^{2}\right]^{\frac{1}{1-q}},
\end{aligned}
$$

where $\sigma$ represents the area of the transverse section of the waveguide. The integral above may be calculated by means of a change of variables, $v=k_{x} x-\omega t$, in such a way to use Eq. (27),

$U \leq \frac{\sigma}{4 \sqrt{\pi}} \frac{\left|\vec{E}_{0}\right|^{2}}{(q-1) k_{x}} \frac{\Gamma\left(\frac{3-q}{2(q-1)}\right)}{\Gamma\left(\frac{1}{q-1}\right)}$,

leading to a finite total energy for $1<q<3$. The upper bound in the above equation diverges in the limit $q \rightarrow 1$. As a typical particular case, one has $U \leq \sigma\left|\vec{E}_{0}\right|^{2} /\left(4 k_{x}\right)$, for $q=2$. Hence, it is plain that the total amount of energy that a spatially localized detector can absorb from a real $q$ plane wave is finite, in contrast to what happens with standard plane waves. These solutions may be useful for describing nonlinear excitations whose shape does not deform in time and could be relevant, e.g., in nonlinear optics and plasma physics.

\section{Conclusions}

We proposed a generalized Lagrangian that leads to a nonlinear extension of the Proca field equation. We discussed some of the main features of this nonlinear field theory, focusing on the existence of exact time-dependent, localized solutions of the $q$-plane wave form. These solutions exhibit soliton-like properties, in the sense of propagating with constant velocity and without changing shape. They have a $q$-plane wave form, which is a generalization of the standard complex exponential plane wave solutions of the linear Proca equation. The $q$-plane waves have properties suggesting that they describe free particles of a finite mass $m$ : they are compatible with the 
celebrated Einstein-Planck-de Broglie connection between frequency, wave number, energy, and momentum, satisfying the relativistic relation $E=p^{2} c^{2}+m^{2} c^{4}$.

In the limit $q \rightarrow 1$, the present nonlinear Proca field theory reduces to the Maxwell linear electrodynamics. We see then that the $q$-deformation associated with the non-extensive formalism turns Proca's linear field theory into its nonlinear generalization, but leaves Maxwell electrodynamics invariant. As already mentioned, for each value $q \neq 1$ the associated nonlinear Proca equation admits $q$-plane wave solutions. In the $q \rightarrow 1$ limit, however, the $q$-plane wave solutions are solutions of Maxwell equations for all $q$.

The nonlinear Proca equation here introduced, together with the nonlinear Dirac and Klein-Gordon equations previously advanced in $[1,7]$, provide the main ingredients of a nonlinear generalization of the basic relativistic field equations for particle dynamics inspired by the $q$-thermostatistical formalism. These equations share a family of exact timedependent solutions: the $q$-plane waves. The present discussion suggests several possible directions of future research, such as to study more complex wave-packet solutions, and to consider interactions. Some progress in these directions has been achieved, in a non-relativistic setting, in the case of a nonlinear Schrödinger equation with a power-law nonlinearity in the kinetic energy term [3-5]. It would be interesting to extend these results to relativistic scenarios. Another relevant issue for future exploration concerns gauge invariance. As happens with the standard linear Proca equation, the present nonlinear Proca equation is not gauge invariant, due to the presence of the mass term. It would be interesting to explore whether this symmetry can be restored by adding more dynamics to the nonlinear Proca field theory, as is done in the liner theory via the Stueckelberg procedure [23]. Any new developments along these lines will be very welcome.

Acknowledgments We thank $\mathrm{C}$. Tsallis for fruitful conversations. The partial financial support from CNPq through Grant 401512/2014-2 and from FAPERJ (Brazilian agencies) is acknowledged.

Open Access This article is distributed under the terms of the Creative Commons Attribution 4.0 International License (http://creativecomm ons.org/licenses/by/4.0/), which permits unrestricted use, distribution, and reproduction in any medium, provided you give appropriate credit to the original author(s) and the source, provide a link to the Creative Commons license, and indicate if changes were made.

Funded by SCOAP ${ }^{3}$.

\section{References}

1. F.D. Nobre, M.A. Rego-Monteiro, C. Tsallis, Phys. Rev. Lett. 106, $140601(2011)$

2. F.D. Nobre, M.A. Rego-Monteiro, C. Tsallis, Europhys. Lett. 97, 41001 (2012)

3. A.R. Plastino, C. Tsallis, J. Math. Phys. 54, 041505 (2013)
4. S. Curilef, A.R. Plastino, A. Plastino, Phys. A 392, 2631-2642 (2013)

5. A.R. Plastino, A.M.C. Souza, F.D. Nobre, C. Tsallis, Phys. Rev. A 90, 062134 (2014)

6. L.G.A. Alves, H.V. Ribeiro, M.A.F. Santos, R.S. Mendes, E.K. Lenzi, Phys. A 429, 35 (2015)

7. M.A. Rego-Monteiro, F.D. Nobre, Nonlinear quantum equations: classical field theory. J. Math. Phys. 54, 103302 (2013)

8. C. Tsallis, Introduction to nonextensive statistical mechanics (Springer, New York, 2009)

9. C. Tsallis, Possible generalization of Boltzmann-Gibbs statistics. J. Stat. Phys. 52, 479 (1988)

10. T.D. Frank, Nonlinear Fokker-Planck equations: fundamentals and applications (Springer, Berlin, 2005)

11. A.R. Plastino, A. Plastino, Phys. A 222, 347 (1995)

12. C. Tsallis, D.J. Bukman, Phys. Rev. E 54, R2197 (1996)

13. V. Schwämmle, F.D. Nobre, E.M.F. Curado, Phys. Rev. E 76, 041123 (2007)

14. J.S. Andrade Jr., G.F.T. da Silva, A.A. Moreira, F.D. Nobre, E.M.F. Curado, Phys. Rev. Lett. 105, 260601 (2010)

15. M.S. Ribeiro, F.D. Nobre, E.M.F. Curado, Phys. Rev. E 85, 021146 (2012)

16. F.D. Nobre, A.M.C. Souza, E.M.F. Curado, Phys. Rev. E 86, 061113 (2012)

17. E.M.F. Curado, A.M.C. Souza, F.D. Nobre, R.F.S. Andrade, Phys. Rev. E 89, 022117 (2014)

18. A. Proca, J. Phys. Radium 7, 347 (1936)

19. W. Greiner, Relativistic quantum mechanics, 3rd edn. (Springer, Berlin, 2000)

20. W. Pauli, Rev. Mod. Phys. 13, 213 (1941)

21. J.D. Jackson, Classical electrodynamics, 3rd edn. (Wiley, New York, 1998)

22. L.C. Tu, J. Luo, G.T. Gillies, Rep. Prog. Phys. 68, 77 (2005)

23. A.S. Goldhaber, M.M. Nieto, Rev. Mod. Phys. 82, 939 (2010)

24. C. Herdeiro, M.O.P. Sampaio, M. Wang, Phys. Rev. D 85, 024005 (2012)

25. G. Dvali, M. Papucci, M.D. Schwartz, Phys. Rev. Lett. 94, 191602 (2005)

26. R. Tomaschitz, Europhys. Lett. 89, 39002 (2010)

27. H. Belich, T. Costa-Soares, M.M. Ferreira Jr., J.A. Helayel-Neto, Eur. Phys. J. C 42, 127 (2005)

28. C. Vuille, J. Ipser, J. Gallagher, Gen. Relativ. Gravit. 34, 689 (2002)

29. R.W. Tucker, C. Wang, Nucl. Phys. B Proc. Suppl. 57, 259 (1997)

30. A.P. Santos, F.I.M. Pereira, R. Silva, J.S. Alcaniz, J. Phys. G Nucl. Part. Phys. 41, 055105 (2014)

31. F.I.M. Pereira, R. Silva, J.S. Alcaniz, Phys. Rev. C 76, 015201 (2007)

32. A. Lavagno, D. Pigato, J. Phys. G NucL. Part. Phys. 39, 125106 (2012)

33. D.P. Menezes, A. Deppman, E. Megas, L.B. Castro, Eur. Phys. J. A 51, 155 (2015)

34. A. Lavagno, D. Pigato, Eur. Phys. J A 47, 52 (2011)

35. A.S. Parvan, Eur. Phys. J. A 51, 108 (2015)

36. A. Deppman, J. Phys. G Nucl. Part. Phys. 41, 055108 (2014)

37. L. Marques, E. Andrade, A. Deppman, Phys. Rev. D 87, 114022 (2013)

38. A. Lavagno, D. Pigato, P. Quarati, J. Phys. G Nucl. Part. Phys. 37, $115102(2010)$

39. M.D. Azmi, J. Cleymans, Eur. Phys. J. C 75, 430 (2015)

40. M. Rybczynski, Z. Wlodarczyk, Eur. Phys. J. C 74, 2785 (2014)

41. T. Wibig, Eur. Phys. J. C 74, 2966 (2014)

42. J. Cleymans, D. Worku, J. Phys. G Nucl. Part. Phys. 39, 025006 (2012)

43. A. Bialas, Phys. Lett. B 747, 190 (2015)

44. J. Cleymans, G. Hamar, P. Levai, S. Wheaton, J. Phys. G Nucl. Part. Phys. 36, 064018 (2009) 
45. W.M. Alberico, A. Lavagno, P. Quarati, Eur. Phys. J. C 12, 499 (2000)

46. C.Y. Wong, G. Wilk, L.J.L. Cirto, C. Tsallis, Phys. Rev. D 91, 114027 (2015)

47. J. Roynek, G. Wilk, J. Phys. G Nucl. Part. Phys. 36, 125108 (2009)

48. J.M. Conroy, H.G. Miller, Phys. Rev. D 78, 054010 (2008)

49. C. Beck, Phys. A 331, 173 (2003)

50. K. Ourabah, M. Tribeche, Ann. Phys. 342, 78 (2014)

51. M.R. Ubriaco, Phys. Lett. A 376, 2899 (2012)
52. E.M.C. Abreu, J.A. Neto, Ann. Phys. 351, 290 (2014)

53. E.M.C. Abreu, J.A. Neto, Phys. Lett. B 727, 524 (2013)

54. A. Lavagno, A.M. Scarfone, P. Narayana Swamy, Eur. Phys. J. C 47, 253 (2006)

55. C. Tsallis, L.J.I. Cirto, Eur. Phys. J. C 73, 2487 (2013)

56. N. Komatsu, S. Kimura, Phys. Rev. D 88, 083534 (2013)

57. E.P. Borges, J. Phys. A 31, 5281 (1998)

58. M. Jauregui, C. Tsallis, J. Math. Phys. 51, 063304 (2010) 